\title{
Serum Level of IL-10 Is Increased in Patients with Endometriosis, and IL-10 Promotes the Growth of Lesions in a Murine Model
}

\author{
Jau-Ling Suen, ${ }^{\dagger \ddagger}$ Yu Chang, ${ }^{\star \S}$ Pu-Rong Chiu, ${ }^{*}$ Tsung-Hua Hsieh,, Edward Hsi, ${ }^{*}{ }^{\star}$ Yu-Chieh Chen, ${ }^{\circledR}$ Yu-Fang Chen, ${ }^{*}$ and \\ Eing-Mei Tsai ${ }^{\star \$ \$}$
}

\begin{abstract}
From the Graduate Institute of Medicine, $*$ the Department of Microbiology, ${ }^{\dagger}$ Faculty of Medicine, College of Medicine, and the Center for Resources, Research and Development, ${ }^{\ddagger}$ Kaohsiung Medical University, Kaohsiung; and the Departments of Obstetrics and Gynecology ${ }^{\S}$ and Medical Research, ${ }^{\natural}$ Kaohsiung Medical University Hospital, Kaohsiung, Taiwan
\end{abstract}

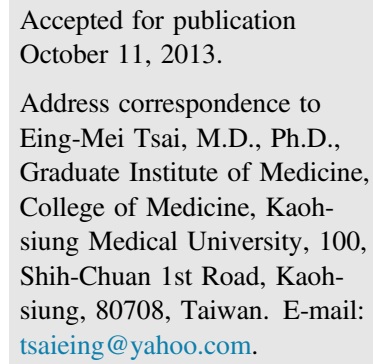

\begin{abstract}
Immune dysregulation may be involved in the development of endometriosis. The anti-inflammatory cytokine IL-10 plays an important role in eliminating unwanted cells and cellular debris in a silent way. We investigated the modulatory role of IL-10 in the development of endometriosis. We observed that the serum level of IL-10 in patients with endometriosis was significantly higher than that in healthy subjects or in control subjects with other gynecological disease. Monocyte-derived dendritic cells acquired from male donors and subsequently conditioned with serum from women with endometriosis exhibited a tolerogenic phenotype, including increased IL-10 production, lower IL-12 secretion, and down-regulation of CD86 and HLA-DR molecules. Depletion of IL-10 activity in a C57BL/6 mouse model of surgically induced endometriosis significantly decreased the size of endometrial lesions. In contrast, IL-10 administration promoted the growth of endometrial lesions in this model. In addition, infiltrated plasmacytoid dendritic cells were the primary IL-10-secreting immune cells in endometrial lesions. Our findings suggest that IL-10 may suppress immunity against endometrial implants, contributing to development of endometriosis. (Am J Pathol 2014, 184: 464-471; http://dx.doi.org/10.1016/j.ajpath.2013.10.023)
\end{abstract}

Endometriosis is a common, benign, and estrogen-dependent gynecological disease characterized by the growth of endometrial cells outside the uterine cavity. The prevalence of endometriosis in the female reproductive years is estimated to be approximately $6 \%$ to $10 \%$. $^{1}$ The pathogenesis of endometriosis is not fully understood. Several theories have been proposed, including retrograde menstruation, celomic metaplasia, and genetic and/or environmental causes. ${ }^{1}$ The most widely accepted theory is retrograde menstruation, with subsequent transplantation and invasion of the peritoneum by shed endometrial fragments. ${ }^{2}$ However, retrograde menstruation alone cannot completely explain the occurrence of endometriosis, because most women (76\% to 90\%) experience some retrograde menstruation, but far fewer women ( $6 \%$ to $10 \%$ ) develop endometriosis. ${ }^{3}$ Accumulating evidence suggests that defective immune surveillance permitting the survival of endometrial tissue after retrograde menstruation may play a crucial role in the genesis and development of endometriosis.

Endometrial tissue in the peritoneal cavity may, if host immunity cannot eliminate it, elicit aberrant inflammation. The anti-inflammatory cytokine IL-10 acts on many types of cells to limit and terminate local inflammation. The removal of apoptotic cells is mediated by dendritic cells (DCs) and macrophages. IL-10-producing macrophages are primarily involved in the silent clearance of early apoptotic cells in which the release of proinflammatory cytokines is prevented. ${ }^{5}$ IL-10 can induce differentiation of DCs that leads to a tolerogenic phenotype, which includes decreased T-cell

Supported by grants NSC-99-2628-B-037-009-MY3, NSC100-2314-B037-043, NSC 102-2628-B-037-011-MY3 from the National Science Council (Taiwan), and by grants KMUH101-1R27, KMUH100-0R24, KMUH 99-9I04, and KMUH 99-9R30 from Kaohsiung Medical University Hospital.

J.-L.S. and Y.C. contributed equally to this work. 
stimulatory activity, increased IL-10 production, and enhanced differentiation of adaptive regulatory $\mathrm{T}$ cells. ${ }^{6} \mathrm{In}$ contrast, late apoptotic cells or necrotic cells may produce danger signals and present self-antigens to DCs, thus activating autoimmune responses and finally leading to the development of autoimmunity, such as occurs in systemic lupus erythematosus. ${ }^{7}$

Endometriosis is a chronic inflammatory disease that is associated with a complex cytokine pattern in the peritoneal fluid; these cytokines include proinflammatory cytokines (IL-1 $\beta$, IL-6, IL-8, and TNF- $\alpha$ ), T-cell-related cytokines (IL-4, IL-12, IL-17, and IFN- $\gamma$ ), and the anti-inflammatory mediator IL-10. ${ }^{4,8}$ Dysregulated adaptive immunity in patients has also been reported, including an imbalance in the activity of helper T cells (Th1, ${ }^{9} \mathrm{Th} 2,{ }^{10} \mathrm{Th} 17^{11}$ ) and of regulatory $\mathrm{T}$ cells, ${ }^{12-14}$ suppressed function of natural killer cells, ${ }^{15}$ increased numbers of neutrophils, ${ }^{16}$ and altered DC function. ${ }^{13,17}$ Thus, dysfunction of natural immunity may lead to inefficient recognition of endometrial antigens, with subsequent inadequate clearance of cellular debris, which then accumulates in the peritoneal cavity after cyclic intraperitoneal microhemorrhages. In the present study, we tested the hypothesis that dysregulation of IL-10 expression contributes to the development of endometriosis.

\section{Materials and Methods}

\section{Study Participants}

Subjects were enrolled after approval of the protocol by the Kaohsiung Medical University Hospital Institutional Review Board. Informed consent was obtained from all participants in accordance with the Declaration of Helsinki. The severity of endometriosis was determined according to the revised American Society of Reproductive Medicine (rASRM) classification in 1996. The endometriosis group consisted of 41 women (mean age, $34.0 \pm 7.1$ years) who underwent surgery for the treatment of advanced endometriosis (rASRM stages III-IV); none of these women had received any medical treatment for at least 3 months before surgery. The disease control group consisted of 26 women (mean age, $36.6 \pm 7.9$ years) who underwent surgery for benign gynecological disease and without evidence of endometriosis as diagnosed during surgery, including absence of endometrial polyps, teratoma, ovarian cysts, and myoma. The healthy control group consisted of 11 women (mean age, $33.1 \pm 3.4$ years) with no gynecological disease. All women in the two control groups had regular menstrual cycles. Clinicodemographic information for the three groups is summarized in Table 1 .

All study participants had visited the outpatient Department of Obstetrics and Gynecology of the Kaohsiung Medical University Hospital. Exclusion criteria were the presence of any autoimmune disease, allergic disease, malignancy, or hepatitis B virus or hepatitis $\mathrm{C}$ virus infection, or any medical treatment or surgery within 3 months before the study-related surgery.

\section{Sample Collection}

Venous blood (3 to $5 \mathrm{~mL}$ ) was drawn from each study participant into a $5-\mathrm{mL}$ sterile syringe and immediately transferred into a sterile clot tube. After the clotting, samples were centrifuged to obtain serum. The serum obtained from each sample was aliquoted and frozen at $-20^{\circ} \mathrm{C}$ until cell culture or cytokine detection.

\section{Generation of Monocyte-Derived DCs}

Peripheral blood mononuclear cells were isolated from male volunteers ( $n=5$; mean age, $25 \pm 3$ years) after informed consent. Circulating $\mathrm{CD} 14^{+}$monocytes were purified using a human CD14 selection kit (STEMCELL Technologies, Vancouver, BC, Canada). The purity of isolated monocytes was $\geq 96 \%$. CD $14^{+}$monocytes were differentiated into DCs in RPMI 1640 medium supplemented with $800 \mathrm{U} / \mathrm{mL}$ recombinant human granulocyte-macrophage colony-stimulating factor (GM-CSF), $500 \mathrm{U} / \mathrm{mL}$ recombinant human IL-4 (R\&D Systems, Minneapolis, MN), $10 \%$ serum from each study subject, 2 $\mathrm{mmol} / \mathrm{L} \mathrm{L}$-glutamine, $0.5 \mathrm{mmol} / \mathrm{L}$ sodium pyruvate, $50 \mu \mathrm{mol} / \mathrm{L}$ nonessential amino acids, $50 \mu \mathrm{mol} / \mathrm{L}$ 2-mercaptoethanol, 10 mmol/L HEPES (Gibco; Life Technologies, Carlsbad, CA), 100 $\mathrm{U} / \mathrm{mL}$ penicillin, and $100 \mu \mathrm{g} / \mathrm{mL}$ streptomycin (Invitrogen; Life Technologies). After 6 days of culture, the monocyte-derived DCs (moDCs) were washed and either left unstimulated or

Table 1 Clinicodemographic Data for Control Subjects and Patients with Endometriosis Enrolled in This Study

\begin{tabular}{|c|c|c|c|c|}
\hline & Healthy control subjects & Disease control subjects & Endometriosis patients & $P$ value* \\
\hline Sample size & $n=11$ & $n=26$ & $n=41$ & \\
\hline Age, years (range) & $33.1 \pm 3.4(28-41)$ & $36.6 \pm 7.9(22-52)$ & $34.0 \pm 7.1(24-46)$ & 0.246 \\
\hline Gravidity (range) & $1.55 \pm 1.21^{\dagger}(0-3)$ & $2.20 \pm 1.73^{\ddagger}(0-7)^{\prime}$ & $0.68 \pm 1.15(0-4)$ & $<0.001$ \\
\hline Parity (range) & $1.36 \pm 1.03^{\S}(0-3)$ & $1.56 \pm 1.12^{\top}(0-4)$ & $0.51 \pm 0.90(0-3)$ & $<0.001$ \\
\hline Weight, kg (range) & $50.6 \pm 5.0(43-58)$ & $57.4 \pm 8.3(40-73)$ & $55.0 \pm 9.7(42-92)$ & 0.104 \\
\hline Height, cm (range) & $162.2 \pm 3.1(158-168)$ & $158.3 \pm 5.1(149.5-168)$ & $160.3 \pm 7.5(145-182.5)$ & 0.193 \\
\hline
\end{tabular}

Data are expressed as means \pm SD.

* Significance was determined with analysis of variance, followed by post hoc Scheffé's multiple comparison test.

${ }^{\dagger} P=0.424$ versus disease control; $P=0.188$ versus endometriosis.

${ }^{\ddagger} P<0.001$ versus endometriosis.

${ }^{\S} P=0.861$ versus disease control; $P=0.047$ versus endometriosis.

$\Upsilon_{P}<0.001$ versus endometriosis. 
stimulated with $1 \mu \mathrm{g} / \mathrm{mL}$ lipopolysaccharide (LPS; SigmaAldrich, St. Louis, MO) for another 24 hours. Cells were harvested for phenotypic analysis using flow cytometry; culture supernatants were used for cytokine pattern determination with ELISA (R\&D Systems).

\section{Flow Cytometry}

The moDCs were stained with the following fluorochromeconjugated monoclonal antibodies (mAbs), or their corresponding isotype controls, purchased from BD Biosciences (San Jose, CA): anti-CD40-FITC (5C3), anti-CD86-PE (2331), anti-CD209-PerCP-Cy5.5 (DCN46), anti-CD11cAPC (B-lym6), anti-CD80-FITC (L307.4), anti-DEC205PerCP (MG38), anti-HLA-DR (L243), and anti-CD83-APC (HB15e). All antibodies were used at optimal concentrations. Cells were analyzed with an LSR II flow cytometer (BD Biosciences) and FlowJo software version 10.0.5 (Tree Star, Ashland, OR).

\section{Mice and the Surgical Endometriosis Model}

All animal studies were conducted in accordance with the Kaohsiung Medical University guidelines for animal use and care (IACUC no. 99123). IL-10 ${ }^{\mathrm{GFP}}$ mice on the C57BL/6 background were obtained from the Jackson Laboratory (Bar Harbor, ME) and were maintained at the Animal Center of Kaohsiung Medical University in a pathogen-free facility. Female control or IL-10 ${ }^{\mathrm{GFP}}$ mice at the age of 6 to 10 weeks were used for surgery.

We established the endometriosis model as described previously, ${ }^{18}$ with some modifications. In brief, the uterine horns from syngeneic female mice were opened longitudinally and removed using a dermal biopsy punch. Intraperitoneal endometrial lesions were surgically induced by suturing four identically sized uterine tissue samples to each side of the abdominal wall. To examine the role of IL-10, we injected each mouse with an anti-IL-10 blocking mAb $(20 \mu \mathrm{g} / \mathrm{mL}, 20$ $\mu \mathrm{L} /$ lesion) (BD Biosciences) or recombinant murine IL-10 (10 or $100 \mathrm{U} / \mathrm{mL}, 20 \mu \mathrm{L} /$ lesion) (PeproTech, Rocky Hill, $\mathrm{NJ})$ into the peritoneum under each transplanted tissue on the right side and corresponding controls on the left side. At days 3,21 , and 28 after surgery, the lesions were collected, and the lesion area was measured using ImageJ version $1.46 \mathrm{r}(\mathrm{NIH}$, Bethesda, MD) image analysis software. For histological analysis, formalin-fixed sections were stained with H\&E. Images were acquired and analyzed with a TissueFAXS cell analysis system (TissueGnostics, Tarzana, CA).

\section{Analysis of IL-10-Secreting Cells in Endometrial Lesions}

To separate single cells from implanted tissues, the endometrial fragments from each mouse were pooled and incubated for 40 minutes in $0.05 \%$ trypsin, $0.53 \mathrm{mmol} / \mathrm{L}$ EDTA, $0.1 \%$ collagenase $\mathrm{D}$, and $150 \mu \mathrm{g} / \mathrm{mL}$ DNase I and were mechanically disrupted with a gentleMACS dissociator (Miltenyi Biotec, Auburn, CA) according to the manufacturer's instructions. Each single-cell suspension was stained with anti-CD45-PECy7 (30-F11), anti-CD11c-APC (N418), anti-PDCA-1-PE (ebio927), anti-CD3-APC (145-2C11), or anti-B220-PE (RA3-6B2) and analyzed with an LSR II flow cytometer (BD Biosciences).

\section{Statistical Analysis}

Differences among the clinical information of study participants were analyzed using analysis of variance followed by a post hoc Scheffé's multiple comparison test. Differences between any two groups in cytokine levels, surface markers, and lesion sizes were analyzed using the nonparametric $U$-test. Differences were considered significant at $P<0.05$.

\section{Results}

No significant differences in age, weight, or height were found among the healthy control subjects, disease control
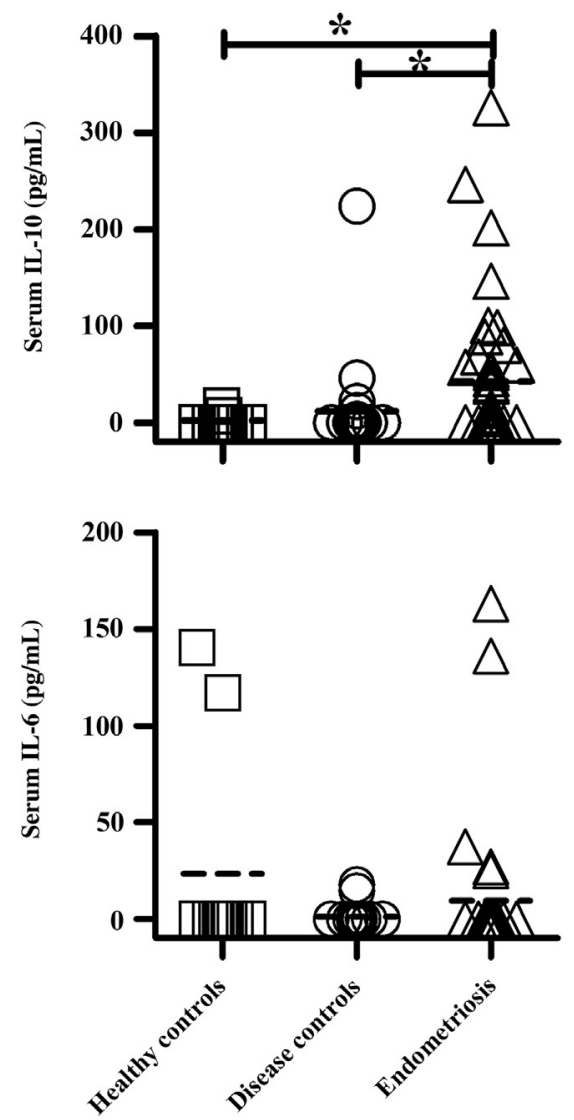

Figure 1 Serum levels of IL-10 are significantly higher in patients with endometriosis than in control subjects. Serum levels of IL-10 and IL-6 were determined using ELISA, with $2 \mathrm{pg} / \mathrm{mL}$ as the limit of detection (ie, the lowest level of quantification). Symbols indicate the cytokine level in a single sample. A horizontal dashed line marks the mean for each group: healthy control subjects $(n=11)$, disease control subjects $(n=26)$, and patients with endometriosis $(n=41)$. ${ }^{*} P<0.05$, nonparametric $U$-test. 
A

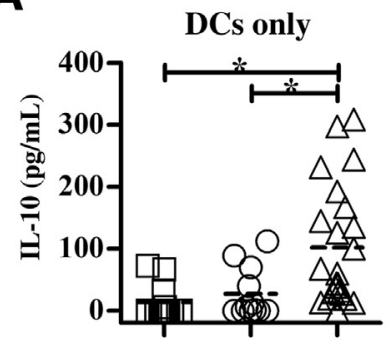

B

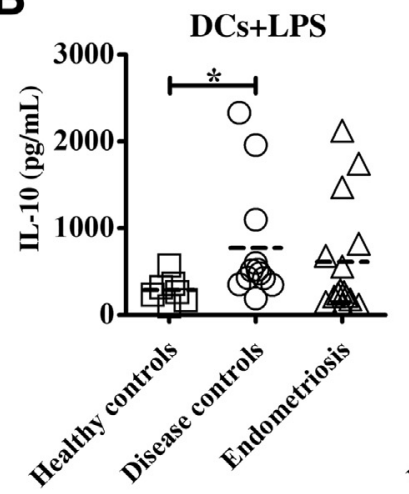

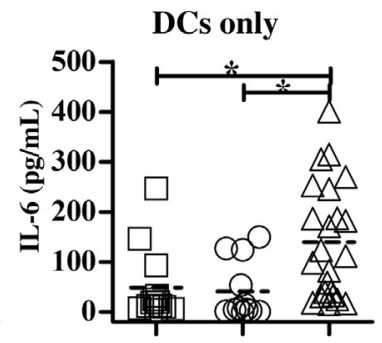
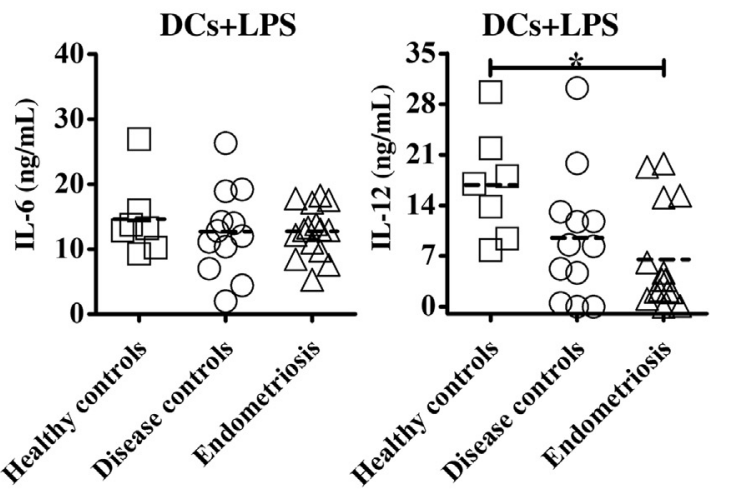

Figure 2 moDCs cultured with serum from patients with endometriosis secrete more of the anti-inflammatory cytokine IL-10 and less of the inflammatory cytokine IL-12. A and B: Purified $\mathrm{CD}_{14}{ }^{+}$monocytes from healthy male donors were cultured for 6 days with GM-CSF, IL-4, and 10\% serum from individuals in the three groups of women. The moDCs were washed and cultured for a further 24 hours with (B) or without LPS (A). Cytokine levels in the supernatant were determined using ELISA, with $4 \mathrm{pg} / \mathrm{mL}$ as the limit of detection. Symbols indicate the cytokine level in a single sample. A horizontal dashed line marks the mean for each group: healthy control subjects $[n=13(\mathbf{A})$ or $7(\mathbf{B})]$, disease control subjects $[(n=12(\mathbf{A}$ and $\mathbf{B})]$, and patients with endometriosis $[n=23$ (A) or $15(\mathbf{B})]$. ${ }^{*} P<0.05$, nonparametric $U$-test. subjects, and patients with endometriosis; as expected, gravidity and parity were much lower in the endometriosis group than in the control group. Low gravidity and low parity are associated with risk of endometriosis. ${ }^{19}$

\section{High IL-10 Levels in Patient Serum}

To examine whether IL-10 overexpression is associated with development of endometriosis, we measured the levels of IL-10, as well as IL-12 and IL-6, in serum of study participants. In contrast to the anti-inflammatory activity of IL-10, IL-12 promotes the differentiation of Th1 cells, which guide immune effectors to clear unwanted cells and debris in an antigen-specific inflammatory process. ${ }^{20}$ IL- 6 is a proinflammatory cytokine with pleiotropic function. ${ }^{21}$ Increased IL-10 levels in serum were observed in patients with endometriosis, but not in disease control subjects or healthy control subjects (Figure 1). Similar levels of IL-6 and undetectable levels of IL-12 were found in all three study groups (data not shown).

\section{Patient Serum-Conditioned moDCs Exhibit a Tolerogenic Phenotype}

To examine whether serum IL-10 exerts suppressive activity, we used DCs cultured in endometriosis patient serum-
A
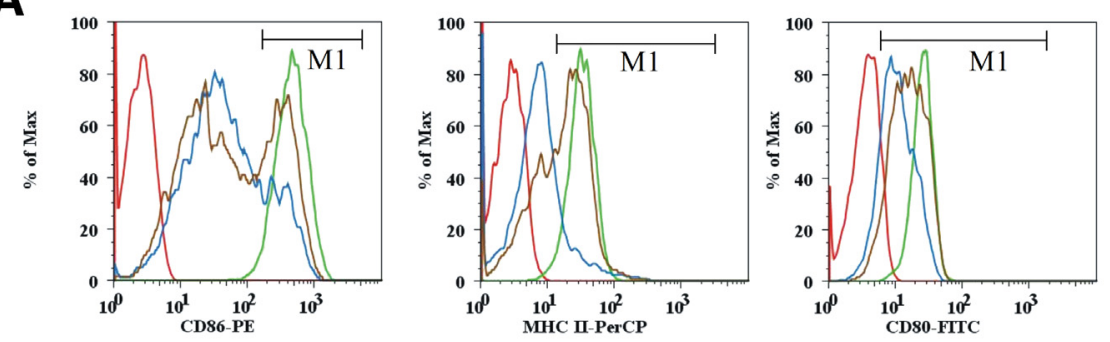

B

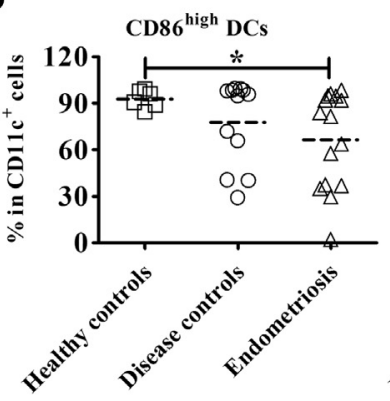

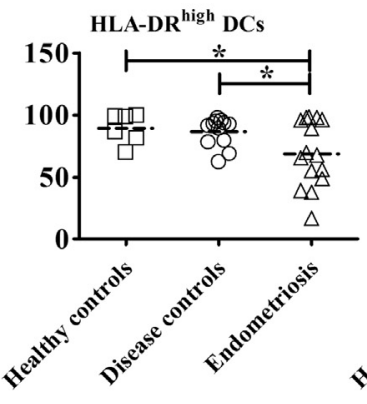

$\mathrm{CD80}^{+} \mathrm{DCs}$

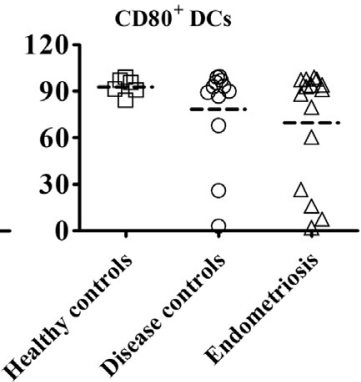

Figure 3 moDCs conditioned with serum from patients with endometriosis exhibit semimature phenotypes in vitro. The moDCs were cultured with serum from individuals in the three groups for 6 days and then stimulated with LPS for 24 hours. Harvested cells were stained with fluorochromelabeled Abs for CD11c, CD80, CD86, and HLA-DR and analyzed using flow cytometry gated for live $\mathrm{CD}_{11 \mathrm{c}^{+}}$cells. A: Representative histograms show surface marker expression on moDCs conditioned with serum from one healthy control subject (green) and two patients with endometriosis (blue and brown); the isotype control is indicated in red. B: Percentage of CD86 $6^{\text {high }}$, HLA-DR ${ }^{\text {high }}$, and $\mathrm{CD} 80^{+}$ moDCs (M1 gates in panel $A$ ) in healthy control subjects $(n=6)$, disease control subjects $(n=$ $12)$, and patients with endometriosis $(n=15)$. ${ }^{*} P<0.05$, nonparametric $U$-test. FITC, fluorescein isothiocyanate; $\mathrm{PE}$, phycoerythrin; PerCP, peridinin chlorophyll protein. 


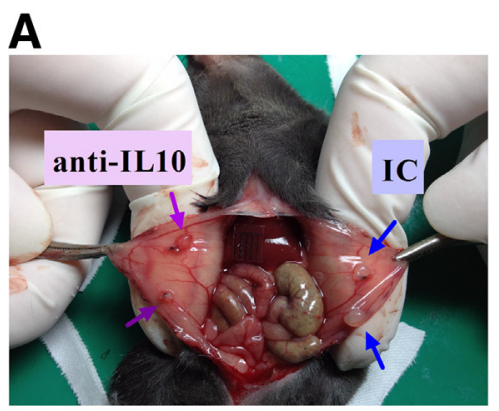

B

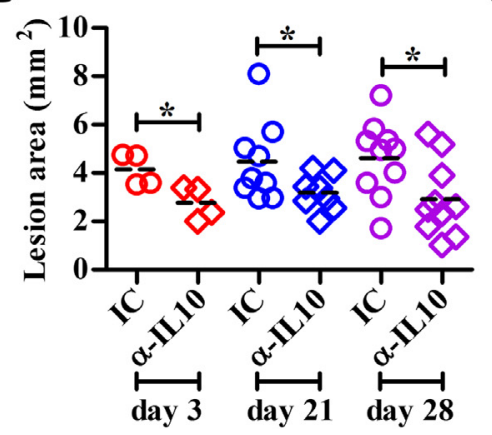

C

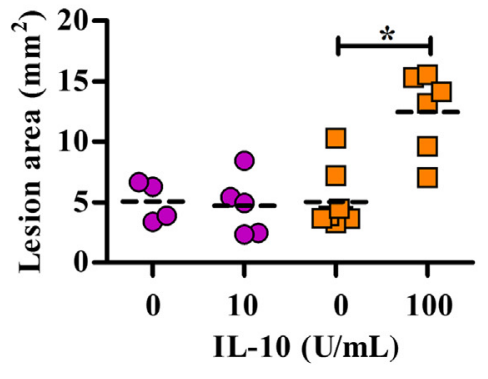

Figure 4 Treatment with a mAb that blocks IL-10 inhibits the growth of endometrial lesions. Four dermal biopsy punches (2 mm each) of uterine horns from C57BL/6 mice were sutured to the four corners of the peritoneal walls in syngeneic naïve mice. A and B: Anti-IL-10 blocking mAb ( $\alpha$-IL10; purple arrows) or isotype control (IC; blue arrows) was locally injected into the tissue at the implantation site; shown is a representative animal treated with the mAbs for 4 weeks (A). Lesion area was calculated in two to five individual mice from two independent experiments (B). C: Recombinant IL-10 or PBS was locally injected into the tissue after the biopsy punches had been sutured to the peritoneal walls in C57BL/6 mice. Lesion area was calculated in two to four individual mice from two independent experiments. A horizontal dashed line marks the mean for each group. ${ }^{*} P<0.05$ versus IC, nonparametric $U$-test.

containing medium, because IL-10 modulates DCs to differentiate into cells with a tolerogenic phenotype. ${ }^{6}$ Given that the differing levels of estrogen during the menstrual cycle significantly affect the maturation and function of DCs, ${ }^{22}$ we used moDCs from healthy male donors, to avoid the possibility that the DCs from female subjects might be in a different state of maturity. We analyzed cytokine levels in supernatants obtained after culturing male monocytes with $10 \%$ serum from study participants during differentiation induced by GM-CSF plus IL-4. The moDCs cultured with serum from patients with endometriosis spontaneously secreted more IL-10 and IL-6 (Figure 2A). The moDCs stimulated with LPS and serum from patients with endometriosis expressed less IL-12 than when serum from healthy control subjects was used (Figure 2B). In contrast, the level of IL-6 expressed by moDCs in the presence of LPS was similar across the three groups. These findings indicate that patient serum-conditioned DCs produced higher levels of the antiinflammatory cytokine IL-10 but lower levels of the inflammatory cytokine IL- 12 .

Next, we analyzed the phenotypic difference in moDCs conditioned with serum from endometriosis patients or control subjects. The moDCs conditioned with serum from healthy control subjects expressed high levels of CD86, HLA-DR (MHC class II molecule), and CD80 in response
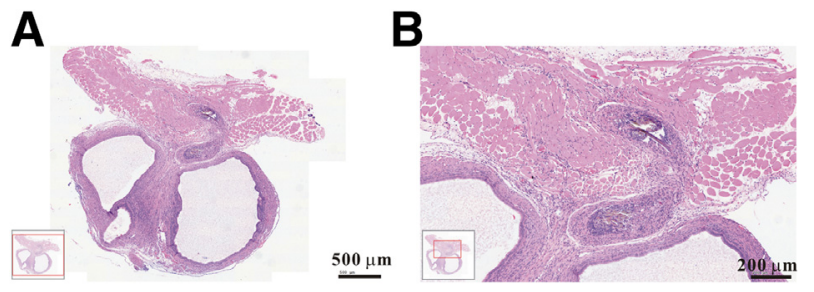

Figure 5 Histological examination of endometrial lesions. Lesions were harvested on day 28 after endometrial surgery and were immediately fixed in formalin. A: Representative section stained with H\&E. B: In an enlarged portion of the same section, the lesion demonstrates typical endometrial features, including glands, stroma, muscles, and infiltrated mononuclear cells. Scale bars: $500 \mu \mathrm{m}$ (A); $200 \mu \mathrm{m}$ (B). to LPS stimulation, representing the mature phenotype of moDCs (Figure 3). In contrast, moDCs conditioned with serum from patients with endometriosis exhibited reduced levels of CD86 and HLA-DR and decreased percentages of CD86 ${ }^{\text {high }}$ and HLA-DR ${ }^{\text {high }}$ moDCs. The levels of other DC-associated molecules, including CD40, CD80, CD83, CD205, and CD209, were similar across the three study groups (data not shown). These findings indicate that patient serum-conditioned moDCs were resistant to LPS-induced maturation, which suggests a tolerogenic property of patient serum-conditioned DCs.

\section{Local IL-10 Activity Enhances Development of Endometrial Lesions}

Next, we examined whether IL-10 is involved in the development of endometriosis, using a mouse model of surgically induced endometriosis. Anti-IL-10 blocking $\mathrm{mAb}$ or its corresponding isotype control was locally injected into the sutured explants in mice that had undergone surgically induced endometriosis. Anti-IL-10 treatment at the beginning of lesion establishment significantly inhibited the growth of endometrial lesions at different time points, from day 3 to day 28 (Figure 4, A and B). In contrast, recombinant IL-10 administration in conjunction with lesion establishment significantly increased the development of endometrial lesions (Figure 4C). These findings suggested that low IL-10 activity may enhance the inflammatory response against the ectopic endometrium, whereas high IL-10 expression may suppress immunity and lead to the development of endometriosis.

\section{Plasmacytoid Dendritic Cells in Endometrial Lesions Comprise the Major IL-10-Producing $\mathrm{CD}_{4} 5^{+}$Cell Subset}

Next, we clarified the type of cell that secretes IL-10 in endometrial lesions. Many different types of immune cells 

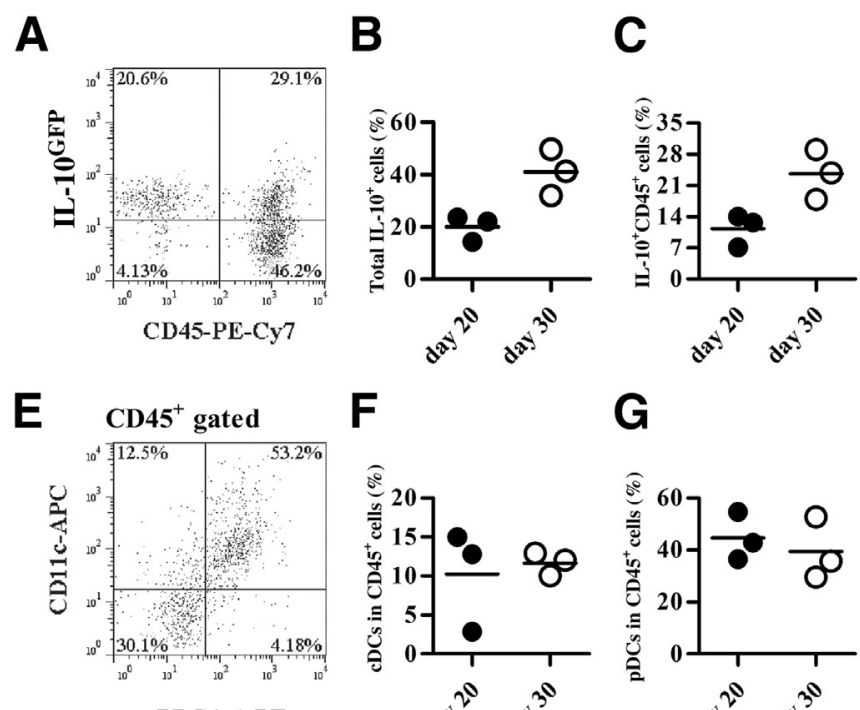

$\mathbf{F}$

\section{G}
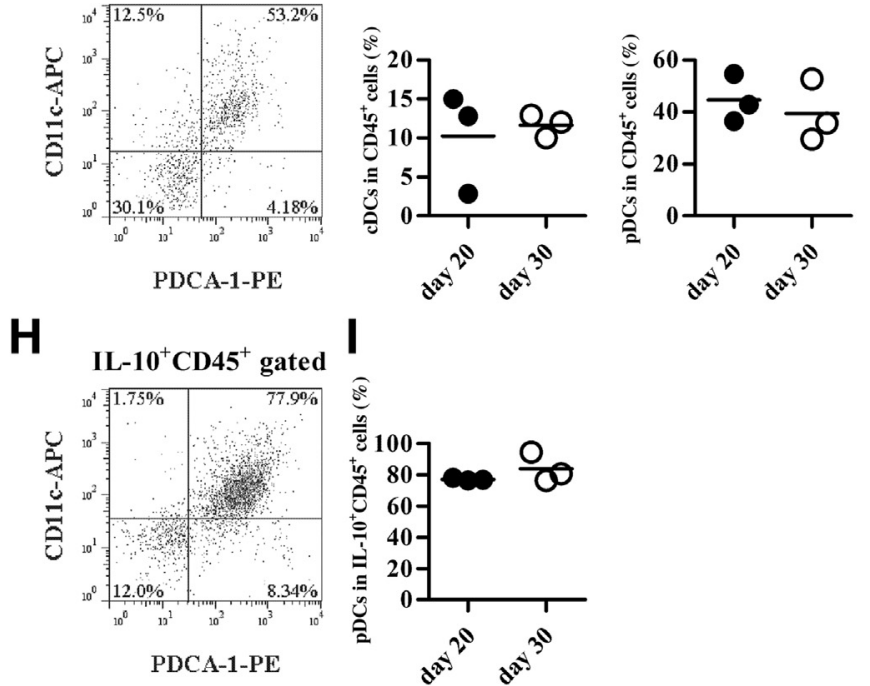

can secrete IL-10 in the context of inflammation, including CD11 $\mathrm{c}^{+}$DCs, Th2 cells, type 1 regulatory $\mathrm{T}$ cells, Foxp ${ }^{+}$ regulatory $\mathrm{T}$ cells, $\mathrm{B}$ cells, and macrophages. ${ }^{23}$ Indeed, we observed that a substantial number of mononuclear cells had infiltrated into endometrial lesions (Figure 5). To easily track and identify IL-10-secreting cells in vivo, we used IL-10 ${ }^{\text {GFP }}$ mice to establish the endometriosis model. At 20 or 30 days after surgery, we used multiparameter flow cytometry to analyze the composition of immune cells in single-cell suspension that had infiltrated into the endometrial lesions. Approximately $20 \%$ and $40 \%$ of cells in the lesions secreted IL-10 at 20 and 30 days after surgery, respectively (Figure 6, A-D). Among the IL-10-secreting cells, approximately half were CD $45^{+}$ immune cells; the remainder were $\mathrm{CD} 45^{-}$nonimmune cells. In addition, $\mathrm{CD} 45^{+}$immune cells contained approximately $10 \%$ conventional DCs (cDCs) $\left(\mathrm{CD} 11 \mathrm{c}^{+} \mathrm{PDCA}-1^{-}\right)$and $40 \%$ plasmacytoid DCs (pDCs) $\left(\mathrm{CD} 11 \mathrm{c}^{+} \mathrm{PDCA}-1^{+}\right)$in the lesions (Figure 6, E-G). Interestingly, among the IL-10-secreting CD $45^{+}$immune cells, approximately $75 \%$ were pDCs at both 20 and 30 days after surgery (Figure 6, H and I). These findings suggested that local IL-10-secreting pDCs, at least in part, play an important role in the development of endometriosis.

\section{Discussion}

In the 1920s, John A. Sampson provided the first theory for the pathogenesis of endometriosis, in terms of retrograde

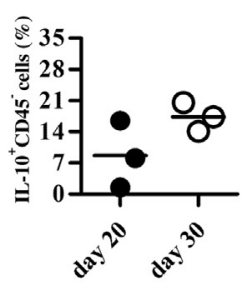

Figure 6 In endometrial tissue, the local IL$10-$ secreting immune cells are mainly pDCs. Dermal punches of uterine horns from IL-10 ${ }^{\mathrm{GFP}}$ mice were sutured to the peritoneal walls of syngeneic naive mice. A, E, and $\mathbf{H}$ : After 20 and 30 days, single cells from the lesions were collected and stained with mAbs specific for CD45, CD11c, and PDCA-1 and analyzed using flow cytometry for expression of CD45 and IL-10 $10^{\text {FFP }}$ in live cells (A) and for CD11C and the expression of PDCA-1 in gated $\mathrm{CD} 45^{+}$cells $(\mathbf{E})$ or gated IL- $10^{\mathrm{GFP}} \mathrm{CD} 45^{+}$cells (H). B-D: Percentage of IL- $10^{+}$subsets (total, $\mathrm{CD} 45^{+}$, and $\mathrm{CD} 45^{-}$) in live cells. $\mathbf{F}$ and $\mathbf{G}$ : Percentage of CDCs (CD11 $\mathrm{c}^{+}$PDCA-1 ${ }^{-}$) (F) and pDCs (CD11 $\mathrm{c}^{+}$PDCA- $\left.{ }^{+}\right)$(G) in $\mathrm{CD}^{+} 5^{+}$immune cells. I: Percentage of pDCs in IL-10 ${ }^{\mathrm{GFP}} \mathrm{CD} 45^{+}$ cells. Symbols indicate the frequency of each subset in individual mice. Data are representative of one of three independent experiments. A horizontal line marks the mean for each time point. APC, allophycocyanin. menstruation. ${ }^{24}$ Decades later, in 1981, Dmowski et $\mathrm{al}^{25}$ were the first to demonstrate deficient cellular immunity in patients with endometriosis. Since then, functional changes have been observed in several immunological components of the peritoneal fluid, as well as in the serum, of women with endometriosis. $^{26}$ The immunity hypothesis suggests that deficient immunity against retrograde endometrial fragments may be involved in the pathophysiology of endometriosis. ${ }^{27}$ Some patients with endometriosis also exhibit autoimmune responses, including increased apoptosis, polyclonal B-cell activation, altered T-cell function, and cell-mediated abnormalities. ${ }^{28}$ It is unclear, however, whether this autoimmune process is part of the etiology or is a secondary response to ectopic endometrial cells.

Previous studies have shown that IL-10 protein level is significantly increased in peritoneal fluid ${ }^{29}$ or medium from cultured peritoneal macrophages $^{30,31}$ of women with endometriosis. In support of such observations, in the present study, increased IL-10 levels were found in serum from patients with endometriosis, and patient serum-conditioned moDCs exhibited a semimature phenotype in response to LPS stimulation, which suggests dysregulated IL-10 activity in endometriosis. In the mouse model of surgically induced endometriosis, blockage of IL-10 activity decreased the size of the endometrial lesions, whereas IL-10 administration enhanced the growth of endometriosis. To our knowledge, this is the first study to demonstrate an adverse effect of IL10 overexpression on the development of endometriosis in a 
mouse model. This is also the first study to identify innate pDCs as IL-10-secreting cells in the microenvironment of endometriosis. These findings suggest that overexpression of IL-10 or high IL-10 activity may suppress cell-mediated immune responses and then promote ectopic implantation of endometrial cells.

Several studies have shown that ILIO gene promoter polymorphisms are associated with the risk of endometriosis. Some studies have shown that the frequency of the $\mathrm{C}$ allele at -819 or -592 in the $I L 10$ promoter is significantly increased in patients with endometriosis, compared with control subjects. ${ }^{32,33}$ Recently, a meta-analysis showed that $I L 10-592$ $\mathrm{A} / \mathrm{C}$ polymorphisms are associated with a significantly increased risk of endometriosis in the Asian population in all genetic models and allele contrasts. ${ }^{34}$ Another study showed that the $\mathrm{T}$ allele at -819 in the $I L 10$ promoter is associated with a risk of endometriosis, and that the $\mathrm{T}$ allele produces lower gene expression than the $\mathrm{C}$ allele. ${ }^{35}$ These conflicting results may be due to the different ethnic origins and different scales in the study populations. In addition, a functional study of promoter polymorphisms should be performed in a microenvironment in the context of endometriosis, because gene regulation occurs in a cell type-specific and tissuespecific manner. ${ }^{36}$ Elucidation of the mechanism underlying how human ILIO promoter polymorphisms and IL-10 protein expression lead to endometriosis requires further detailed investigation.

In the present study, we found that an abundance of IL$10-$ secreting pDCs (IL- $\left.10^{\mathrm{GFP}} \mathrm{CD} 11 \mathrm{c}^{+} \mathrm{PDCA}-1^{+}\right)$infiltrate into the endometrial lesions. This observation is in accord with previous findings showing that infiltrated $\mathrm{CD} 11 \mathrm{c}^{+} \mathrm{DCs}$ exhibit an immature phenotype (MHC class II $^{\text {low }}$ ). The infiltrated CD $11 \mathrm{c}^{+}$DCs express VEGFR2 and are associated with increased angiogenesis. ${ }^{18}$ However, it is unclear from that report whether the infiltrating $\mathrm{CD} 11 \mathrm{c}^{+} \mathrm{DCs}$ were $\mathrm{pDCs}$ $\left(\mathrm{PDCA}-1^{+}\right)$or $\mathrm{cDCs}\left(\mathrm{PDCA}-1^{-}\right)$. Because IL-10 may modulate vascular activity at the maternal-fetal interface, ${ }^{37,38}$ we speculate that infiltrating IL-10-secreting pDCs may participate in the development of endometriosis, at least in part, by promoting angiogenesis or suppressing anti-self responses in the lesions. In support of this idea, depletion of IL-10 activity inhibited lesion development, whereas enhancement of IL-10 levels increased the size of lesions (Figure 4). Elucidation of the detailed mechanism involved in the $\mathrm{pDC}-\mathrm{IL}-10$ axis in the pathogenesis of endometriosis requires further investigation.

IL-10 plays a critical role in regulating physiological responses, including dampening immunity to pathogens in the context of infection, maintenance of immune tolerance, ${ }^{39}$ and controlling a successful pregnancy. ${ }^{40} \mathrm{IL}-10$ is secreted by a wide variety of cell types, including both immune $\left(\mathrm{CD} 45^{+}\right)$and nonimmune (CD45 $\left.{ }^{-}\right)$. In accord, we observed that approximately $50 \%$ of IL-10-secreting cells were immune cells, with the remainder being nonimmune cells (Figure 6). To our knowledge, this is the first report of IL-10 production in local pDCs in the context of endometriosis.
Because others have reported that pDCs do not secrete IL10 in response to microbial products, ${ }^{41}$ our novel observation suggests that $\mathrm{pDCs}$ control endometrial inflammation in response to endogenous danger signals, such as local apoptotic cells. The roles of pDCs in the anti-inflammatory response and the pathogenesis of endometriosis should be explored in greater depth.

\section{Acknowledgments}

We thank the Center for Resources, Research and Development of Kaohsiung Medical University for providing the LSR II and TissueFAXS system.

J.-L.S. and E.M.T. conceived and designed experiments; Y.C., P.L.C., T.H.H., E.H., Y.C.C., and Y.F.C. performed experiments and analyzed data; all authors were involved in writing the paper and had final approval of the submitted and published versions.

\section{References}

1. Giudice LC, Kao LC: Endometriosis. Lancet 2004, 364:1789-1799

2. D'Hooghe TM, Debrock S: Endometriosis, retrograde menstruation and peritoneal inflammation in women and in baboons. Hum Reprod Update 2002, 8:84-88

3. Witz CA: Interleukin-6: another piece of the endometriosis-cytokine puzzle. Fertil Steril 2000, 73:212-214

4. Lebovic DI, Mueller MD, Taylor RN: Immunobiology of endometriosis. Fertil Steril 2001, 75:1-10

5. Xu W, Roos A, Schlagwein N, Woltman AM, Daha MR, van Kooten C: IL-10-producing macrophages preferentially clear early apoptotic cells. Blood 2006, 107:4930-4937

6. Rutella S, Danese S, Leone G: Tolerogenic dendritic cells: cytokine modulation comes of age. Blood 2006, 108:1435-1440

7. Fransen JH, van der Vlag J, Ruben J, Adema GJ, Berden JH, Hilbrands LB: The role of dendritic cells in the pathogenesis of systemic lupus erythematosus. Arthritis Res Ther 2010, 12:207-214

8. Gazvani R, Templeton A: Peritoneal environment, cytokines and angiogenesis in the pathophysiology of endometriosis. Reproduction 2002, 123:217-226

9. Mier-Cabrera J, Jiménez-Zamudio L, García-Latorre E, Cruz-Orozco O, Hernández-Guerrero C: Quantitative and qualitative peritoneal immune profiles. T-cell apoptosis and oxidative stress-associated characteristics in women with minimal and mild endometriosis. BJOG 2011, 118: $6-16$

10. Podgaec S, Abrao MS, Dias JA Jr, Rizzo LV, de Oliveira RM, Baracat EC: Endometriosis: an inflammatory disease with a Th2 immune response component. Hum Reprod 2007, 22:1373-1379

11. Hirata T, Osuga Y, Takamura M, Kodama A, Hirota Y, Koga K, Yoshino O, Harada M, Takemura Y, Yano T, Taketani Y: Recruitment of CCR6-expressing Th17 cells by CCL 20 secreted from IL-1 beta-, TNF-alpha-, and IL-17A-stimulated endometriotic stromal cells. Endocrinology 2010, 151:5468-5476

12. Berbic M, Hey-Cunningham AJ, Ng C, Tokushige N, Ganewatta S, Markham R, Russell P, Fraser IS: The role of Foxp3+ regulatory T-cells in endometriosis: a potential controlling mechanism for a complex, chronic immunological condition. Hum Reprod 2010, 25: 900-907

13. Berbic M, Fraser IS: Regulatory T cells and other leukocytes in the pathogenesis of endometriosis. J Reprod Immunol 2010, 88:149-155

14. Basta P, Majka M, Jozwicki W, Lukaszewska E, Knafel A, Grabiec M, Stasienko E, Wicherek L: The frequency of CD25+CD4+ and 
FOXP3 + regulatory $\mathrm{T}$ cells in ectopic endometrium and ectopic decidua. Reprod Biol Endocrinol 2010, 8:116

15. Eidukaite A, Siaurys A, Tamosiunas V: Aberrant expression of CD95 and CD69 molecules among CD56 cells in women with endometriosis. Am J Reprod Immunol 2006, 55:276-281

16. Tariverdian N, Siedentopf F, Rücke M, Blois SM, Klapp BF, Kentenich H, Arck PC: Intraperitoneal immune cell status in infertile women with and without endometriosis. J Reprod Immunol 2009, 80: 80-90

17. Schulke L, Berbic M, Manconi F, Tokushige N, Markham R, Fraser IS: Dendritic cell populations in the eutopic and ectopic endometrium of women with endometriosis. Hum Reprod 2009, 24:1695-1703

18. Fainaru O, Adini A, Benny O, Adini I, Short S, Bazinet L, Nakai K, Pravda E, Hornstein MD, D'Amato RJ, Folkman J: Dendritic cells support angiogenesis and promote lesion growth in a murine model of endometriosis. FASEB J 2008, 22:522-529

19. Hediger ML, Hartnett HJ, Louis GM: Association of endometriosis with body size and figure. Fertil Steril 2005, 84:1366-1374

20. Kim S, Elkon KB, Ma X: Transcriptional suppression of interleukin-12 gene expression following phagocytosis of apoptotic cells. Immunity 2004, 21:643-653

21. Jawa RS, Anillo S, Huntoon K, Baumann H, Kulaylat M: Analytic review: interleukin-6 in surgery, trauma, and critical care: part I: basic science. J Intensive Care Med 2011, 26:3-12

22. Carreras E, Turner S, Frank MB, Knowlton N, Osban J, Centola M, Park CG, Simmons A, Alberola-Ila J, Kovats S: Estrogen receptor signaling promotes dendritic cell differentiation by increasing expression of the transcription factor IRF4. Blood 2010, 115:238-246

23. Hedrich CM, Bream JH: Cell type-specific regulation of IL-10 expression in inflammation and disease. Immunol Res 2010, 47: 185-206

24. Sampson JA: Metastatic or embolic endometriosis, due to the menstrual dissemination of endometrial tissue into the venous circulation. Am J Pathol 1927, 3:93-110.43

25. Dmowski WP, Steele RW, Baker GF: Deficient cellular immunity in endometriosis. Am J Obstet Gynecol 1981, 141:377-383

26. Wu MY, Ho HN: The role of cytokines in endometriosis. Am J Reprod Immunol 2003, 49:285-296

27. Barcz E, Kaminski P, Marianowski L: Role of cytokines in pathogenesis of endometriosis. Med Sci Monit 2000, 6:1042-1046

28. Eisenberg VH, Zolti M, Soriano D: Is there an association between autoimmunity and endometriosis? Autoimmun Rev 2012, 11:806-814

29. Ho HN, Wu MY, Chao KH, Chen CD, Chen SU, Yang YS: Peritoneal interleukin-10 increases with decrease in activated CD4+
T lymphocytes in women with endometriosis. Hum Reprod 1997, 12: $2528-2533$

30. Wu MY, Ho HN, Chen SU, Chao KH, Chen CD, Yang YS: Increase in the production of interleukin-6, interleukin-10, and interleukin-12 by lipopolysaccharide-stimulated peritoneal macrophages from women with endometriosis. Am J Reprod Immunol 1999, 41:106-111

31. Rana N, Braun DP, House R, Gebel H, Rotman C, Dmowski WP: Basal and stimulated secretion of cytokines by peritoneal macrophages in women with endometriosis. Fertil Steril 1996, 65:925-930

32. Zhang X, Hei P, Deng L, Lin J: Interleukin-10 gene promoter polymorphisms and their protein production in peritoneal fluid in patients with endometriosis. Mol Hum Reprod 2007, 13:135-140

33. Xie J, Wang S, He B, Pan Y, Li Y, Zeng Q, Jiang H, Chen J: Association of estrogen receptor alpha and interleukin-10 gene polymorphisms with endometriosis in a Chinese population. Fertil Steril 2009, 92:54-60

34. Fan W, Li S, Chen Q, Huang Z, Ma Q, Xiao Z: Association between interleukin-10 promoter polymorphisms and endometriosis: a metaanalysis. Gene 2013, 515:49-55

35. Juo SH, Wu R, Lin CS, Wu MT, Lee JN, Tsai EM: A functional promoter polymorphism in interleukin-10 gene influences susceptibility to endometriosis. Fertil Steril 2009, 92:1228-1233

36. Zaritsky LA, Dery A, Leong WY, Gama L, Clements JE: Tissuespecific interferon alpha subtype response to SIV infection in brain, spleen, and lung. J Interferon Cytokine Res 2013, 33:24-33

37. Tinsley JH, South S, Chiasson VL, Mitchell BM: Interleukin-10 reduces inflammation, endothelial dysfunction, and blood pressure in hypertensive pregnant rats. Am J Physiol Regul Integr Comp Physiol 2010, 298:R713-R719

38. Tewari N, Kalkunte S, Murray DW, Sharma S: The water channel aquaporin 1 is a novel molecular target of polychlorinated biphenyls for in utero anomalies. J Biol Chem 2009, 284:15224-15232

39. Saraiva M, O'Garra A: The regulation of IL-10 production by immune cells. Nat Rev Immunol 2010, 10:170-181

40. Hanna N, Hanna I, Hleb M, Wagner E, Dougherty J, Balkundi D, Padbury J, Sharma S: Gestational age-dependent expression of IL-10 and its receptor in human placental tissues and isolated cytotrophoblasts. J Immunol 2000, 164:5721-5728

41. Boonstra A, Rajsbaum R, Holman M, Marques R, Asselin-Paturel C, Pereira JP, Bates EE, Akira S, Vieira P, Liu YJ, Trinchieri G, O'Garra A: Macrophages and myeloid dendritic cells, but not plasmacytoid dendritic cells, produce IL-10 in response to MyD88- and TRIF-dependent TLR signals, and TLR-independent signals. J Immunol 2006, 177:7551-7558 